\title{
Myths and Truths of Nitinol Mechanics: Elasticity and Tension- Compression Asymmetry
}

\author{
Ashley N. Bucsek ${ }^{1} \cdot$ Harshad M. Paranjape $^{1} \cdot$ Aaron P. Stebner $^{1}$
}

Published online: 29 August 2016

(C) ASM International 2016

\begin{abstract}
Two prevalent myths of Nitinol mechanics are examined: (1) Martensite is more compliant than austenite; (2) Texture-free Nitinol polycrystals do not exhibit tension-compression asymmetry. By reviewing existing literature, the following truths are revealed: (1) Martensite crystals may be more compliant, equally stiff, or stiffer than austenite crystals, depending on the orientation of the applied load. The Young's Modulus of polycrystalline Nitinol is not a fixed number-it changes with both processing and in operando deformations. Nitinol martensite prefers to behave stiffer under compressive loads and more compliant under tensile loads. (2) Inelastic Nitinol martensite deformation in and of itself is asymmetric, even for texture-free polycrystals. Texture-free Nitinol polycrystals also exhibit tension-compression transformation asymmetry.
\end{abstract}

Keywords Nickel-titanium $\cdot$ Texture $\cdot$ Anisotropy $\cdot$ Shape memory alloy

\section{Introduction}

While shape memory behaviors were first observed in the 1940s and 1950s in gold-cadmium and indium-thallium [1-3], it is the work of William Buehler and colleagues on Nickel-Titanium at the Naval Ordinance Laboratory (i.e., "Nitinol") that provided the foundation for the modern shape memory alloy (SMA) industry. Nitinol showed

Aaron P. Stebner

astebner@mines.edu

1 Department of Mechanical Engineering, Colorado School of Mines, Golden, CO 80401, USA extraordinary functional behaviors-the shape memory effect, actuation, and superelasticity $[4,5]$, as well as practical engineering properties-relatively low homologous temperatures in engineering environments, high strength and stiffness, outstanding corrosion resistance, and favorable reaction to lubrications [6,7]. These beyond shape memory characteristics distinguished Nitinol from other SMAs and established it as the industry standard material.

Functional behaviors of Nitinol arise from a solid-state transformation between cubic austenite and monoclinic martensite phases, which is now a standard subject of SMA texts (e.g., [8-10]). The mechanics of single austenite lattices transforming to single martensite lattices, free of any type of defect, are largely agreed upon and understood. However, Nitinol materials are rarely defect-free, even in single-crystal form. Furthermore, Nitinol materials used in industry (1) are polycrystalline, (2) have severe amounts of cold work introduced to them during processing, and (3) contain precipitates-both $\mathrm{Ni}_{4} \mathrm{Ti}_{3}$ particles intentionally introduced to strengthen the materials and/or tune transformation temperatures, as well as undesirable oxide and/or carbide inclusions that are inherent to melting practices [11]. Because of these different microstructural features, the Nitinol materials that have enabled new technologies exhibit macroscopic mechanics that are far from the theoretical single-crystal case. For this reason, it was difficult to understand the causal relationship between theoretical single-crystal mechanics and macroscopic mechanical responses. As a result, admittedly over-simplified models and explanations were created that likened polycrystalline Nitinol to traditional, better-understood elastic-plastic alloys. Somewhere along the way, the underlying assumptions in making these simplifications were forgotten or ignored by many in the Nitinol industry. Many myths 
were born as a result of these models being taken out of context. Over the past few decades, research has collectively shed light on many of the true mechanics of Nitinol, as well as what we do not yet know. In the remainder of the article, we survey two of the more prominent myths of Nitinol mechanics under the spotlight of truths elucidated through modern research.

\section{Myth 1: Nitinol Martensite is More Compliant than Austenite}

By the 1990s, measurements of Nitinol materials had led to the textbook understanding that the Young's modulus of martensite was much lower than that of austenite: 20-50 GPa for martensite versus 40-90 for austenite (e.g., $[8,12])$. With the benefit of hindsight, there are several immediate indications that these measurements were circumstantial. First, as Liu et al. point out in [13, 14], these modulus values are on the order of soft metals like aluminum and copper (the martensite values even softer), but the melting temperature and ultimate strength of Nitinol are both more comparable to carbon steels, which exhibit a Young's Modulus of $\sim 200 \mathrm{GPa}$-over twice as stiff. Second, the Young's Modulus fundamentally results from the strength of chemical bonds. Because the austenite and martensite unit cells have similar ordering and negligible volume change, one would expect the moduli between the phases to be similar. Finally, the range of the reported moduli is too large to be attributed to experimental variation; thus, it is indicative of a larger misunderstanding of the meaning and circumstances of the measurements.

Despite all of these clues, this myth continues to permeate our industry due to the convenience it affords modelers. It allows engineers to assume that each phase is a separate linear-elastic material, each with its own Young's Modulus $(E)$. One may then compute the transformation strain by calculating the elastic strain $(\varepsilon)$ of the austenite using Hooke's law $(\sigma=E \varepsilon)$ and subtracting it from the martensite elastic strain under some stress $(\sigma)$. For example, if the austenite were modeled with a modulus of $60 \mathrm{GPa}$ and the martensite with $20 \mathrm{GPa}$ for an application where the SMA experienced a constant load of $200 \mathrm{MPa}$ load, such a calculation would tell the engineer to expect $0.7 \%$ transformation strain when switching between phases.

It is easy to understand why such a model is desirable and popular. Most engineers learn Hooke's law, and this approach allows us to teach engineers a method to treat the complex behaviors of Nitinol components using models they already know. Furthermore, the calculations are easily made by hand or with simple spreadsheets. Hence, it is no surprise that as the limitations of the simplest of such modeling approaches were exploited in the 1980s, modified models evolved from this same philosophy. Some treated martensite as a material that followed a piece-wise continuous elastic behavior defined by two or three different moduli, while others simply added an offset to the martensite response to account for transformation/reorientation plateaus; i.e., a non-zero intercept to the linearelastic response.

The "compliant martensite myth" is reasonably founded. In fact, these assumptions are accurate for specially processed NiTi materials, such as highly worked pieces of wire loaded in uniaxial tension, as shown in Fig. 1a. In the early 2000s, however, researchers used specialized techniques such as ultrasonic spectroscopy and in situ neutron diffraction to show that, under other conditions, the Young's Modulus of martensite appears stiffer than that of austenite [15-17]. For a period of time, the pendulum swung in the other direction and some believed that martensite was always stiffer than austenite (see Fig. 1c), and the "apparent modulus" as in Fig. 1a, was an artifact of an ever-present persistence of martensite reorientation (i.e., twinning and detwinning) [13]. This latter viewpoint was again founded in reason; for differently processed Nitinol materials used in specific conditions, such as unworked rods cut from fully annealed extrusions and ingots, martensite reorients under the smallest of loads$10 \mathrm{MPa}$ applied effective stress or less. Thus, there is no true elastic regime in its stress-strain response. Furthermore, the stress-strain responses during initial loading may be non-linear, as shown in Fig. 1b. In considering such circumstances where the "compliant martensite myth" breaks down, the aforementioned engineering models predict compressive transformation strain for tensile loads and vice versa - clearly non-plausible behaviors! Still, the lack of a universal understanding of the stiffness of martensite that could explain all of the modulus measurements (as in Fig. 1) inhibited the development of more physically correct models. For some time, the Nitinol community was divided with two camps: stiff versus compliant martensite.

Fortunately, this split inspired Wagner and Windl and also Hatcher, Kontsevoi, and Freeman to study the problem of monoclinic single-crystal elasticity using atomistic theory $[18,19]$. These researchers realized that the community lacked a fundamental understanding of bond strengths in martensite crystal structures, and such understanding had the potential to lead to universal understanding of martensite elasticity (in addition to other First Principles phenomena we do not discuss here). While the exact numbers reported by each group of researchers depend strongly on the assumptions and boundary conditions used for their simulations, they all agreed on one fundamental finding: The truth is martensite crystals may be more 


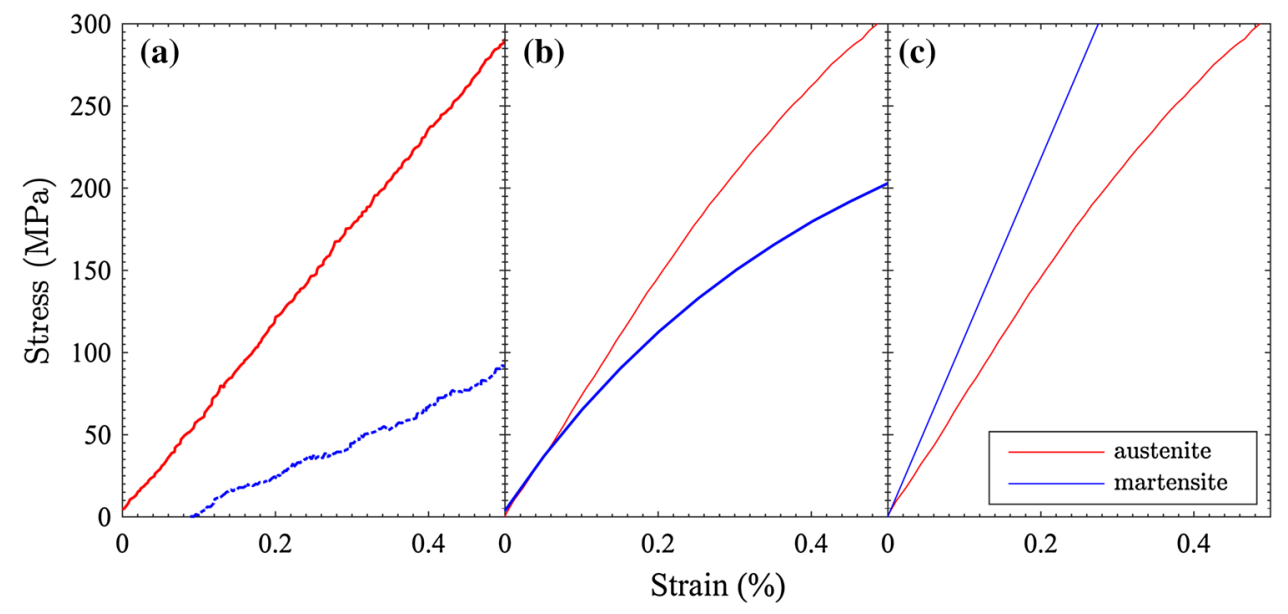

Fig. 1 a Martensite appears more compliant than austenite (highly worked \& textured Nitinol wire data from [22] is shown), b randomtexture, hot extruded martensite [29] appears initially as stiff as austenite [27], and $\mathbf{c}$ random-texture, as-cast martensite appears stiffer than austenite. b Both responses shown are measured from samples

compliant, equally stiff, or stiffer than austenite crystals, depending on the orientation of the loading direction with respect to the unit cells. Because monoclinic crystals are of much lower symmetry, their elastic anisotropy is much greater. Therefore, the most compliant monoclinic orientations are more compliant than their cubic counterparts, the stiffest monoclinic orientations are stiffer than their cubic counterparts, and other orientations exhibit nearly identical stiffness.

An additional insight into the "compliant martensite myth" may be realized by considering that in crystals, stronger or stiffer bonds hold atoms more closely together. Thus, at first order, the map of the orientation dependence of monoclinic Young's modulus given in Fig. 2e is indirectly and approximately a map of the orientation dependence of the bond lengths that dominate the elastic behavior of Nitinol martensite. Now if crystals are given the opportunity to reorient during a deformation, as it has long been known Nitinol martensite will do [20, 21], the principal of energy minimization dictates that orientations of shortest bond lengths are preferred in compression, while longest bond lengths are preferred in tension. ${ }^{1}$ Thus, it is not surprising that in situ diffraction experiments have revealed that preferred martensite textures for tension versus compression loading of Nitinol differ in accordance with the Young's Modulus trends together with the principal of elastic energy minimization. In tension (Fig. 2a), martensite prefers textures near (120) (Fig. 2c), which orient the more compliant, longer bonds with the load directions (Fig. 2e), while in compression, stiff, short-

\footnotetext{
${ }^{1}$ More exactly it is closest packed vs. least close packed planes of atoms, but bond lengths of crystal structures are easier for most engineers to visualize and an appropriate analogy.
}

taken from the exact same hot-extruded rods, even though reports are made by different authors. $\mathbf{c}$ The martensite response is plotted using the reported value of $109 \mathrm{GPa}$ from neutron diffraction measurements of Rajagopalan et al. [16] and the austenite reponse is the same as in b, from Benafan et al. [27]

bonded (101) planes align with loads (Fig. 2d) [22]. Indeed, in situ diffraction data have shown that for many Nitinol materials martensite texture evolves with thermomechanical loading, and consequently so does the Young's Modulus [23-25]. Thus, additional Nitinol elasticity truths have been realized: The Young's Modulus of polycrystalline Nitinol is not a fixed number-it changes with both processing and in operando deformations. Nitinol martensite prefers to behave stiffer under compressive loads and more compliant under tensile loads. While experiments have shown that austenite textures may also evolve [26, 27], because the anisotropy is not as extreme for cubic lattices [28], fluctuations of the austenite elastic modulus during thermomechanical loadings are not as dramatic. This is true, even considering the known anelastic fluctuations in austenite modulus as the transformation temperatures are approached, a subject we do not delve into here, but which has been discussed at length in the literature (e.g., [11], known as "precursor phenomena").

While First Principles calculations of Nitinol monoclinic elastic constants elucidated vastly improved, universally applicable physical understanding of Nitinol elasticity, they have yet to be fully verified via experimental measurements. Five of the 13 independent monoclinic elastic constants were measured using in situ neutron diffraction in [29]. The results suggest that the Wagner and Windl calculations are closer to the properties exhibited by real Nitinol martensites, though they are consistently too stiff, even when accounting for (relatively large) experimental uncertainty. This finding is not surprising, considering that the atomistic calculations are made at absolute zero, ignoring thermal vibrations of atoms, which inherently 

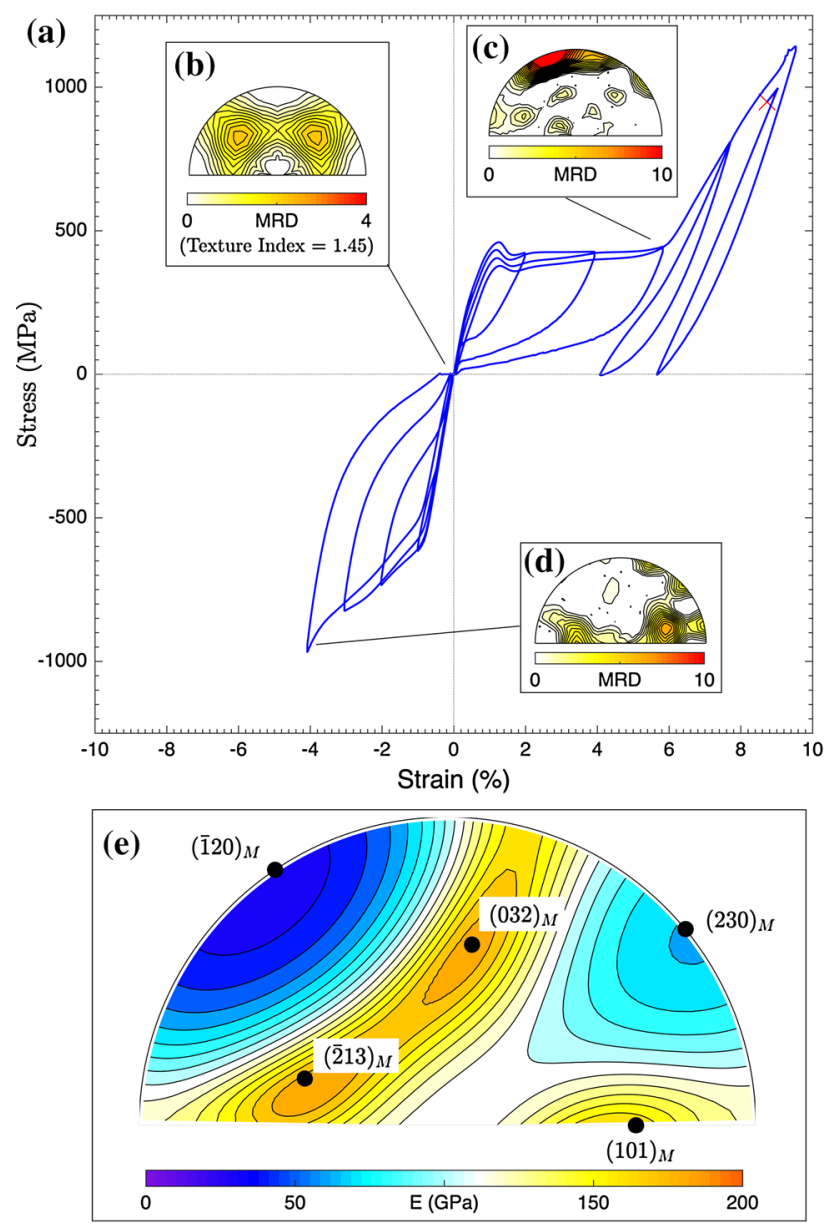

Fig. 2 a Ex situ stress-strain responses of superelastic samples tested using the same techniques, instrumentation, and sample preparation as reported in $[49,55]$ are shown, together with $\mathbf{b}$ the initial austenite load-direction inverse pole figure, $\mathbf{c}$ the tensile load-direction martensite inverse pole figure of the material at $6 \%$ strain, and d the compression load-direction martensite inverse pole figure measured of the material at $-4.25 \%$ strain, all measured in Multiples of Random Distribution (MRD). e Calculations of the orientationdependence of single monoclinic crystal Young's modulus $(E)$ using the Wagner and Windl elastic constants [18] are plotted on an inverse pole figure, with near-minima and maxima orientations labeled

make atomic bonds more compliant. Furthermore, mesoscopic martensite stiffnesses will depend on the martensite variants and twin systems that are able to form under the constraints of austenite-martensite interfaces. Wang and Sehitoglu establish a procedure for calculating the stiffnesses of "Habit Plane Variants" (HPVs), martensite twin pairs that are compatible against an austenite interface according to the Phenomenological Theory of Martensite, using the theoretical single-crystal elastic constants [30]. Such calculations are essential to the long line of micromechanical models that use HPVs as the fundamental martensite microstructure building block, following after the work of Patoor et al. [31]. However, experimental measurement of all 13 Nitinol monoclinic elastic remains an open challenge to our community. Thus, a quantified understanding of monoclinic elasticity is still strongly desired. Our best understanding today results in the (lack of) certainty depicted in Fig. 3, considering the differences between both the square and circle markers (calculations vs. measurements), as well as the errorbars on the circle markers (the reported uncertainty in the experimental measurements). As Wang and Sehitoglu emphasized, this quantified understanding is critical to our ability to properly calculate and model a wide range of SMA phenomena [30]. One final point toward more quantitatively correct measurements of martensite modulus-it has been shown that the initial unloading of martensite (either thermally stable or stress-induced martensite) is a more accurate means to macroscopically measure the martensite modulus than using the initial loading response [23, 29]. Loading responses are riddled by the addition of twinning, phase transformations, and plasticity in all but a very few special instances. Again, because of these things, the martensite modulus is also dynamic during loading, further complicating the acquisition of a scalar value for a model.

\section{Myth 2: Texture-Free Nitinol Polycrystals Exhibit Tension-Compression Symmetry}

This myth was born from understanding plasticity in traditional elastic-plastic metals and alloys, especially those with cubic crystal structures. More specifically, texture-free ductile metals exhibit tension-compression symmetry in small-tomoderate plastic deformations as a result of the relatively high symmetry of both crystal structures and the driving forces (e.g., applied stress) required to activate slip systems [32]. It is easy to imagine how Nitinol was initially likened to traditional ductile metals with its simple cubic austenite phase and apparently ductile inelastic deformations. However, the monoclinic martensite phase of Nitinol is of very low symmetry. As a result, it exhibits a large number of twinning modes (12 or more) as well as at least one slip system $[10,22,33-35]$. The monoclinic twinning modes are highly asymmetric, to the extent that given a single grain orientation; only some modes will produce tensile strain, and others compressive strain, and the number of favorable modes and driving forces needed for each tension and compression are not equal [22]. Thus, the truth is: Inelastic Nitinol martensite deformation in and of itself is asymmetric, even for texturefree polycrystals, as shown in Fig. 4. The reasons for larger plateau strains and less hardening in tension versus compression in this figure are: (1) a higher activity of martensite slip in compression (which produces hardening), (2) fewer active twinning systems in compression, and (3) the favorable compression twinning systems realize less twinning strain [22]. 


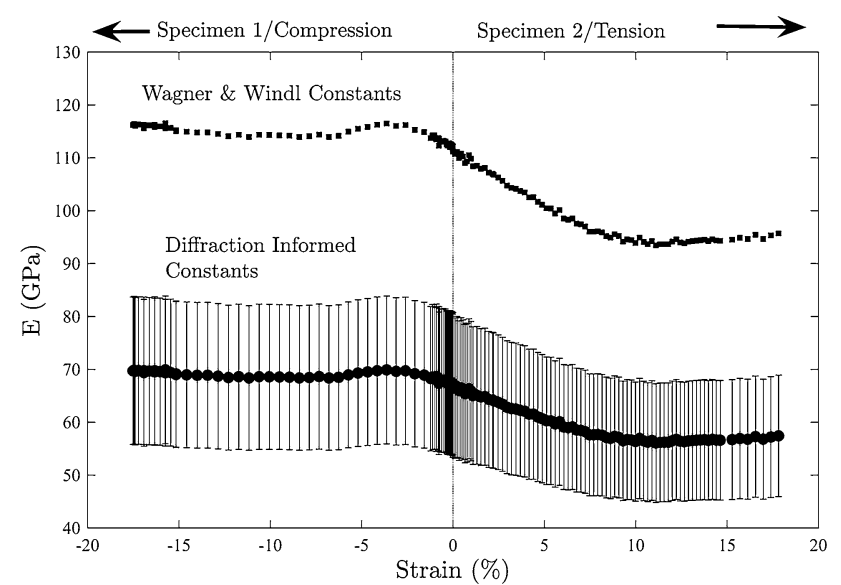

Fig. 3 A depiction of the certainty of current understanding of martensite Young's modulus $(E)$ is shown-note the large errorbars, and even bigger differences between calculations and experimentsthere is still much room for improvement. Calculations of $\mathrm{E}$ made using Wagner \& Windl elastic constants and the neutron diffraction data measured of polycrystals by Stebner et al. [23] are shown with square markers (after Fig. 6 of [23], the Specimen 1, 2 labels follow that work). New to this article, these calculations are then adjusted according to the differences between Wagner and Windl calculations and elastic constants measured using in situ neutron diffraction as reported in [29] (circle markers). The errorbars represent the reported uncertainty in the neutron diffraction measured moduli values in this latter work

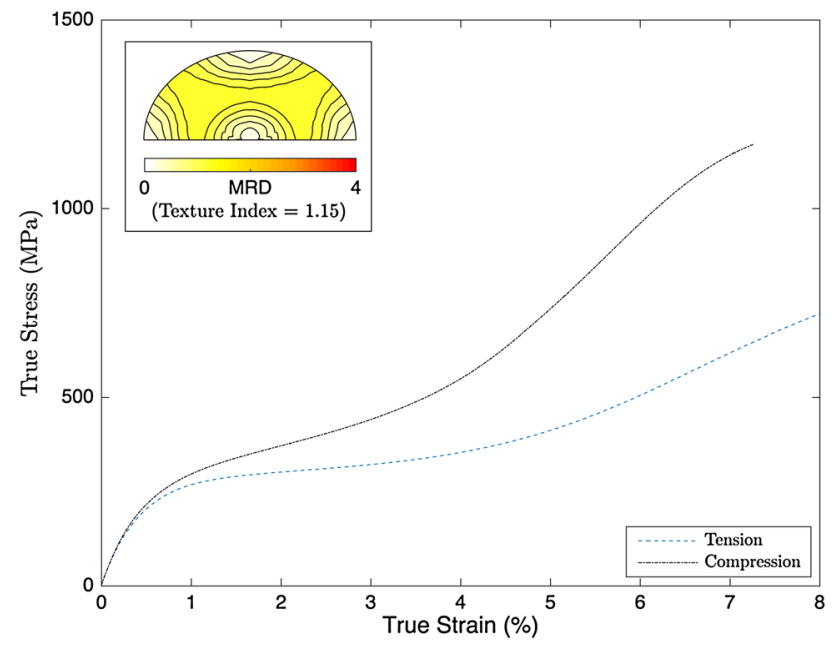

Fig. 4 Tension and compression stress-strain curves are shown for (near) random-textured Nitinol martensite, as indicated in the inset, which shows the initial austenite load-direction inverse pole figure. These original data are ex situ loading curves performed at the same time and using the same techniques and sample preparation as the in situ experiments described in [22, 42]

Still, most Nitinol applications today make use of phase transformation; i.e., superelasticity and actuation. Thus, we also desire an understanding of tension-compression asymmetry of phase transformations in Nitinol, which is not explained solely by inelastic deformations of the monoclinic martensite phase. However, conceptualizing the foundation of transformation asymmetry is as simple as considering the change between austenite and martensite crystal structures. Asymmetry resulting from the structural changes that occur as a result of phase transformations in SMAs has been studied since their discovery in the 1950 s [36], and periodically "rediscovered" since then. In Nitinol, the cubic austenite lattice parameter is on the order of $3.01 \AA$ [28], while the monoclinic martensite unit cell edge lengths are approximately $2.89,4.12$, and $4.62 \AA$ [33]. However, the edges of the primitive cubic unit cell do not directly correspond to the edges of the primitive monoclinic unit cell, but rather we consider transformation from a cell in the cubic structure that has edge lengths of approximately $3.01,4.25$, and $4.25 \AA$ (see [10, p. 52]). Calculating the engineering strain along the cell edges that results from transforming from this cell to the aforementioned monoclinic cell, the axial strains along the sides of the structures are approximately $-4.0,-3.1$, and $+8.0 \%$, respectively. Clearly the tensile transformation strain is larger than the compressive strains. In Fig. 5a, we have plotted the difference in the magnitude of the maximum tensile and compressive transformation strains ( $\left.\left|\varepsilon_{\text {transformation }}^{\text {tension }}\right|-\left|\varepsilon_{\text {transformation }}^{\text {compression }}\right|\right)$ for all orientations of parent cubic crystals transforming to a single monoclinic crystal (calculated according to [10, 37]), and it is evident that for all except a few orientations near (001) Austenite, the tensile strains are larger. The Clausius-Clapeyron equation, as summarized in greater detail by Liu and Yang [14], states:

$\frac{\mathrm{d} \sigma}{\mathrm{d} T}=\frac{\rho \Delta S}{\varepsilon_{t r}}$,

where $\sigma$ is stress, $T$ is temperature, $\rho$ is density, $\Delta S$ is the change in entropy from the phase transformation (and temperature change), and $\varepsilon_{t r}$ is the transformation strain. Thus at a fixed temperature, it takes less stress (i.e., driving force) to induce a transformation in a material with larger transformation strain through this inverse proportionality. Hence the asymmetry in transformation strains naturally gives rise to the inverse asymmetry in transformation stresses: more stress is required to transform Nitinol crystals in compression than tension for most orientations.

While the single-crystal foundation of tension-compression asymmetry is well established and easy to understand, the nature of polycrystals is more complicated. In fact, if we move to consider transformation from an austenite grain to HPVs instead of single monoclinic crystals, and then repeat the previous exercise of calculating the maximum tension and compression strains and taking the difference in magnitudes $\left(\left|\varepsilon_{\text {transformation }}^{\text {tension }}\right|-\left|\varepsilon_{\text {transformation }}^{\text {compression }}\right|\right)$ over all orientations, there 


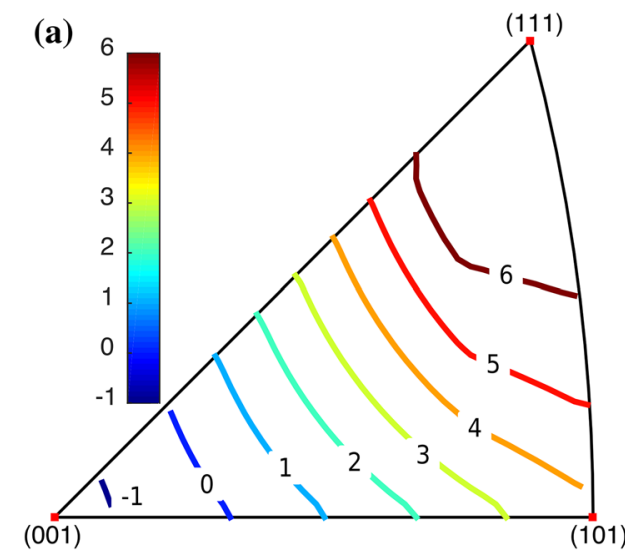

Fig. 5 Inverse pole figures showing the orientation dependence of maximum tension-compression transformation strain differences $\left(\left|\varepsilon_{\text {transformation }}^{\text {tension }}\right|-\left|\varepsilon_{\text {transformation }}^{\text {compression }}\right|\right)$. a The maximum transformation strain along the loading direction is calculated assuming a single-crystal austenite to single martensite correspondence variant (CV)

are now slightly more orientations that exhibit larger compressive strains than tensile strains (Fig. 5b, note that over half of the stereographic map shows the compressive strains to be larger). This calculation clearly explains why models that consider only transformation from austenite grains to HPVs suggest that random Nitinol polycrystals will show tension-compression asymmetry that is opposite what is traditionally observed in materials-smaller transformation stresses and larger transformation strains in compression [38, 39]—while polycrystalline models based on single crystal mechanics show the opposite trend [40-45]. In addition to these concepts based on single crystal mechanics, other factors that can contribute to asymmetric microstructural evolution in polycrystals are the incomplete phase transformation based on the crystal orientation of the grain, complex interactions between grain neighborhoods and the resultant multi-axial stress states as well as a potential asymmetric interaction between transformation and slip. Recent empirical investigations discussed below attempt to address these aspects.

A single unified source of experimental validation as to which theory is more accurate does not currently exist in the open literature. This lack of data results from very few documented uses of texture-free Nitinol. The closest comparison we are able to make is to consider the compression results of Vaidyanthan et al. [46], who made material that had an austenite texture index of approximately 0.90 (1.00 would be perfectly random) and observed approximately $-3.5 \%$ superelastic transformation strain and $-500 \mathrm{MPa}$ transformation stress. That compressive behavior can then be compared to tension actuation responses of the material shown in Fig. 4, which has an initial austenite texture index of 1.15 [47] and

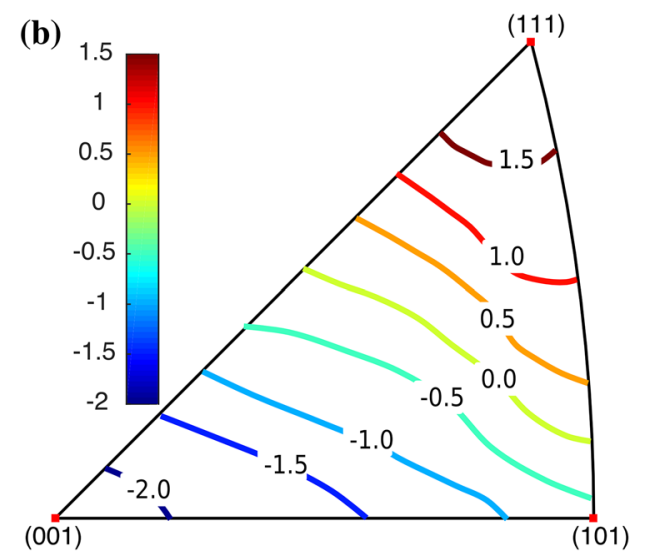

transformation. b Stress-induced transformation from an austenite single crystal to the most favorable martensite habit plane variant (HPV) is considered. These calculations reveal that for certain austenite orientations close to ( $\left.\begin{array}{lll}0 & 0 & 1\end{array}\right)$, the most favorable $\mathrm{CV}$ or HPV both produce a larger magnitude of transformation strain compression versus tension

exhibits a maximum transformation strain in actuation of $4.3 \%$ under $200 \mathrm{MPa}$ load [48]. In an email correspondence at the time of writing this article, Dr. O. Benafan of NASA indicated that in data not yet published by their research group, they have found that the latter material shows a maximum actuation transformation strain of $-4.1 \%$ under $-300 \mathrm{MPa}$ load in compression testing analogous to the tension tests published in [48]. These comparisons indicate that the overall random polycrystal behavior qualitatively mimics the trend shown by calculations made with single-crystal mechanics-larger tension strains under smaller stresses. This finding is consistent with recent experimental observations that suggest that HPV-based models predict initiation, but not total transformation mechanics [49-51]. Hence an opportunity exists for modeling efforts that include transformation to CVs as well as HPVs to capture the influence of such mixed stressinduced microstructures on tension-compression asymmetry. Such full-field models would be able to capture other empirically observed phenomena like partially transformed grains [52], and the influences of intergranular constraints and coupling with plasticity. Still, in totality, all approaches show that the truth is: random-textured Nitinol polycrystals do exhibit tension-compression transformation asymmetry. Processing texture may further skew this inherent asymmetry, as is the case for wires, tubes, and rods that are readily reported upon in existing literature. This includes the mechanical responses of somewhat textured Nitinol rods (Fig. 2e), which exhibit over $5.5 \%$ transformation strain in tension. Processed textures of Nitinol are usually skewed to (111) and (110) components, as in Fig. 2e, thus following the transformation strain trends shown in Fig. 5a, the Nitinol that is used in industry 
typically exhibits greater tension-compression asymmetry skewed toward more transformation strain at lower transformation stresses in tension than texture-free material.

\section{Conclusion}

Because real Nitinol materials are textured polycrystals that undergo complex thermomechanical processing, they display macroscopic responses that are incredibly varied and usually different from single-crystal theory. For this reason, the link between single-crystal theory and real material behavior was not understood for several decades. Even as more accurate depictions of Nitinol mechanics were being published, models were slow to follow and these myths persisted. However, recent research has elucidated the underlying truths of Nitinol elasticity and tension-compression asymmetry. These truths can be understood through the physics of single-crystal mechanics with proper extension to polycrystals. To this day, however, although the truths are no longer in question, many Nitinol engineers and researchers still hold the myths closely because of their history and simplicity.

One final remark is that understanding the mechanics of Nitinol plasticity, including asymmetry, is still incomplete due to difficulty deconvoluting it from transformation behaviors. Models have shown that the asymmetry of the transformation alone is enough to make plasticity appear asymmetric, even in models that assume a constant, scalar slip stress for plastic flow (e.g., [53, 54]). A more complete physical understanding of plasticity in Nitinol and its role in tension-compression asymmetry is at the forefront of many current research efforts.

Acknowledgments We thank Dr. O. Benafan for sharing the raw data used to create the austenite responses in Fig. 1b, c, and also the email communication of the compression actuation responses stated in Myth 2. A.B. acknowledges the support of NSF Fellowship DGE1057607; H.P. the support of DOE-BES Grant DE-SC0010594; and A.S. the support of NSF-CAREER \#1454668 from CMMI-MoMS.

\section{References}

1. Chang LC, Read TA (1951) Plastic deformation and diffusionless phase changes in metals-The gold-cadmium beta-phase. Am Inst Min Metall Eng 191(1):47-52

2. Chang L-C (1951) Atomic displacements and crystallographic mechanisms in diffusionless transformation of gold-cadium single crystals containing 47.5 atomic per cent cadmium. Acta Crystallogr 4(4):320-324

3. Basinski Z, Christian J (1954) Experiments on the martensitic transformation in single crystals of indium-thallium alloys. Acta Metall 2(1):148-166

4. Buehler WJ, Gilfrich JV, Wiley RC (1963) Effect of low-temperature phase changes on the mechanical properties of alloys near composition TiNi. J Appl Phys 34(5):1475-1477
5. Buehler WJ, Wang FE (1968) A summary of recent research on the Nitinol alloys and their potential application in ocean engineering. Ocean Eng 1(1):105-120

6. Buehler WJ, Wiley RC (1962) TiNi-ductile intermetallic compound. Trans Am Soc Met 55:269-276

7. Buehler WJ (1963) Intermetallic compound based materials for structural applications. In: US Naval Ordinance Laboratory Silver Springs, Maryland, The seventh navy science symposium: solution to navy problems through advanced technology, vol 14, pp 15-16

8. Duerig TW, Melton KN, Stockel D, Wayman CM (1990) Engineering aspects of shape memory alloys. Butterworth-Heinemann, Boston

9. Otsuka K, Wayman CM (1998) Shape memory materials. Cambridge University Press, Cambridge

10. Bhattacharya K (2003) Microstructure of martensite: why it forms and how it gives rise to the shape memory effect. Oxford University Press, Oxford

11. Otsuka K, Ren X (2005) Physical metallurgy of Ti-Ni-based shape memory alloys. Prog Mater Sci 50(5):511-678

12. Hodgson DE, Ming W, Biermann RJ (1990) "Shape Memory Alloys", in Metals Handbook, vol 2, 10th edn. ASM International, Materials Park, pp 897-902

13. Liu Y, Xiang H (1998) Apparent modulus of elasticity of nearequiatomic NiTi. J Alloys Compd 270:154-159

14. Liu Y, Yang H (1999) The concern of elasticity in stress-induced martensitic transformation in NiTi. Mater Sci Eng A 260(1-2):240-245

15. Ren X, Miura N, Zhang J, Otsuka K, Tanaka K, Koiwa M, Suzuki T, Chumlyakov YI, Asai M (2001) A comparative study of elastic constants of Ti-Ni-based alloys prior to martensitic transformation. Mater Sci Eng A 312(1-2):196-206

16. Rajagopalan S, Little AL, Bourke MAM, Vaidyanathan R (2005) Elastic modulus of shape-memory NiTi from in situ neutron diffraction during macroscopic loading, instrumented indentation, and extensometry. Appl Phys Lett 86(8):081901

17. Sehitoglu H, Karaman I, Anderson R, Zhang X, Gall K, Maier HJ, Chumlyakov Y (2000) Compressive response of NiTi single crystals. Acta Mater 48:3311-3326

18. Wagner MF-X, Windl W (2008) Lattice stability, elastic constants and macroscopic moduli of NiTi martensites from first principles. Acta Mater 56(20):6232-6245

19. Hatcher N, Kontsevoi OY, Freeman AJ (2009) Role of elastic and shear stabilities in the martensitic transformation path of NiTi. Phys Rev B 80(14):144203

20. Liu Y, Xie Z, Humbeeck JV, Delaey L (1998) Asymmetry of stress-strain curves under tension and compression for NiTi shape memory alloys. Acta Mater 46(12):4325-4338

21. Saburi T, Nenno S, Aaronson H, Laughiin D, Sekerka R, Wayman C (1981) Solid to solid phase transformations. Metals Society of AIME, Warrendale, p 1455

22. Stebner A, Vogel S, Noebe R, Sisneros T, Clausen B, Brown D, Garg A, Brinson L (2013) Micromechanical quantification of elastic, twinning, and slip strain partitioning exhibited by polycrystalline, monoclinic nickel-titanium during large uniaxial deformations measured via in situ neutron diffraction. J Mech Phys Solids 61(11):2302-2330

23. Stebner A, Brown D, Brinson L (2013) Young's modulus evolution and texture-based elastic-inelastic strain partitioning during large uniaxial deformations of monoclinic nickel-titanium. Acta Mater 61(6):1944-1956

24. Cai S, Schaffer JE, Ren Y, Yu C (2013) Texture evolution during nitinol martensite detwinning and phase transformation. Appl Phys Lett 103(24):241909

25. Šittner P, Heller L, Pilch J, Curfs C, Alonso T, Favier D (2014) Young's modulus of austenite and martensite phases in superelastic NiTi wires. J Mater Eng Perform 23(7):2303-2314 
26. Bowers ML, Gao Y, Yang L, Gaydosh DJ, De Graef M, Noebe RD, Wang Y, Mills MJ (2015) Austenite grain refinement during load-biased thermal cycling of a Ni49.9Ti50.1 shape memory alloy. Acta Mater 91:318-329

27. Benafan O, Noebe RD, Padula SA, Garg A, Clausen B, Vogel S, Vaidyanathan R (2013) Temperature dependent deformation of the B2 austenite phase of a NiTi shape memory alloy. Int J Plast 51:103-121

28. Mercier O, Melton KN, Gremaud G, Hägi J (1980) Single-crystal elastic constants of the equiatomic NiTi alloy near the martensitic transformation. J Appl Phys 51(3):1833-1834

29. Stebner AP, Brown DW, Brinson LC (2013) Measurement of elastic constants of monoclinic nickel-titanium and validation of first principles calculations. Appl Phys Lett 102(21):211908

30. Wang J, Sehitoglu H (2014) Martensite modulus dilemma in monoclinic NiTi-theory and experiments. Int J Plast 61:17-31

31. Patoor E, Eberhardt A, Berveiller M (1998) Micromechanical modeling of shape memory alloys behavior. Arch Mech 40:775-794

32. Kocks UF, Tome CN, Wenk H-R (1998) Texture and anisotropy. Cambridge University Press, Cambridge

33. Kudoh Y, Tokonami M, Miyazaki S, Otsuka K (1985) Crystal structure of the martensite in Ti-49.2 at.\% $\mathrm{Ni}$ alloy analyzed by the single crystal X-ray diffraction method. Acta Metall 33(11):2049-2056

34. Nishida M, Ii S, Kitamura K, Furukawa T, Chiba A, Hara T, Hiraga K (1998) New deformation twinning mode of B19' martensite in Ti-Ni shape memory alloy. Scr Mater 39(12):1749-1754

35. Karaman I, Kulkarni A, Luo Z (2005) Transformation behaviour and unusual twinning in a NiTi shape memory alloy ausformed using equal channel angular extrusion. Philos Mag 85(16):1729-1745

36. Burkart MW (1953) Diffusionless phase change in the indiumthallium system. Am Inst Min Metall Eng 227:1515-1524

37. Miyazaki S, Kimura S, Otsuka K, Suzuki Y (1984) The habit plane and transformation strains associated with the martensitic transformation in Ti-Ni single crystals. Scr Metall 18(9):883-888

38. Thamburaja P, Anand L (2001) Polycrystalline shape-memory materials: effect of crystallographic texture. J Mech Phys Solids 49(4):709-737

39. Gall K, Sehitoglu H, Chumlyakov YI, Kireeva IV (1999) Tension-compression asymmetry of the stress-strain response in aged single crystal and polycrystalline NiTi. Acta Mater 47(4):1203-1217

40. Bhattacharya K, Kohn RV (1996) Symmetry, texture and the recoverable strain of shape-memory polycrystals. Acta Mater 44(2):529-542

41. Shu YC, Bhattacharya K (1998) The influence of texture on the shape-memory effect in polycrystals. Acta Mater 46(15):5457-5473

42. Stebner AP, Bigelow GS, Yang J, Shukla DP, Saghaian SM, Rogers R, Garg A, Karaca HE, Chumlyakov Y, Bhattacharya K et al (2014) Transformation strains and temperatures of a nickeltitanium-hafnium high temperature shape memory alloy. Acta Mater 76:40-53

43. Bucsek AN, Hudish GA, Bigelow GS, Noebe RD, Stebner AP (2016) Composition, compatibility, and the functional performances of ternary nitix high-temperature shape memory alloys. Shape Mem Superelast 2(1):62-79

44. Šittner P, Novák V (2000) Anisotropy of martensitic transformations in modeling of shape memory alloy polycrystals. Int $\mathrm{J}$ Plast 16(10-11):1243-1268

45. Novak V, Sittner P (2004) Micromechanics modelling of NiTi polycrystalline aggregates transforming under tension and compression stress. Mater Sci Eng 378:490-498

46. Vaidyanathan R, Bourke M, Dunand D (2001) Texture, strain, and phase-fraction measurements during mechanical cycling in superelastic NiTi. Metall Mater Trans A 32(13):777-786

47. Stebner AP (2012) Partitioning of elastic, transformation, and plastic strains exhibited by shape-memory nickel-titanium through modeling and neutron diffraction. Ph.D. thesis, Northwestern University

48. Santo Padula I, Qiu S, Gaydosh D, Noebe R, Bigelow G, Garg A, Vaidyanathan R (2012) Effect of upper-cycle temperature on the load-biased, strain-temperature response of NiTi. Metall Mater Trans A 43(12):4610-4621

49. Stebner AP, Paranjape HM, Clausen B, Brinson LC, Pelton AR (2015) In Situ Neutron Diffraction Studies of Large Monotonic Deformations of Superelastic Nitinol. Shape Mem Superelast 1(2):252-267

50. Kimiecik M, Jones J, Daly S (2015) Grain orientation dependence of martensitic phase transformation in polycrystalline shape memory alloys. Acta Mater 94:214-223

51. Kimiecik M, Jones JW, Daly S (2016) The effect of microstructure on stress-induced martensitic transformation under cyclic loading in the SMA Nickel-Titanium. J Mech Phys Solids 89:16-30

52. Kimiecik M, Jones JW, Daly S (2013) Quantitative Studies of microstructural phase transformation in Nickel-Titanium. Mater Lett 95:25-29

53. Richards AW, Lebensohn RA, Bhattacharya K (2013) Interplay of martensitic phase transformation and plastic slip in polycrystals. Acta Mater 61(12):4384-4397

54. Stebner A, Bhattacharya K (2014) Micromechanics inspired, phenomenological model of fully coupled plasticity, phase transformation, and martensite reorientation in shape memory alloys. In: Bajaj A, Zavattieri P, Koslowski M, Siegmund T (eds) Proceedings of the society of engineering science 51st annual technical meeting, Purdue University Libraries Scholarly Publishing Services, West Lafayette, October 1-3 2014. http://docs. lib.purdue.edu/ses2014/mss/cppt/3

55. Pelton AR, Clausen B, Stebner AP (2015) In Situ Neutron Diffraction Studies of Increasing Tension Strains of Superelastic Nitinol. Shape Mem Superelast 1(3):375-386 